Latorre-Román, P.A.; Lloris-Ogallar, E.; Salas-Sánchez, J.; García-Pinillos, F. (2020). Association between Executive Function, Intellectual Maturity and Physical Fitness in Preschool Children. Revista Internacional de Medicina y Ciencias de la Actividad Física y el Deporte vol. 20 (79) pp. 471-485 Http://cdeporte.rediris.es/revista/revista79/artasociacion1176.htm

DOI: http://doi.org/10.15366/rimcafd2020.79.006

\title{
ORIGINAL
}

\section{ASOCIACIÓN ENTRE FUNCIÓN EJECUTIVA, MADUREZ INTELECTUAL Y CONDICIÓN FÍSICA EN NIÑOS PREESCOLARES}

\section{ASSOCIATION BETWEEN EXECUTIVE FUNCTION, INTELLECTUAL MATURITY AND PHYSICAL FITNESS IN PRESCHOOL CHILDREN}

\author{
Latorre-Román, P.A. ; Lloris-Ogallar, E. ${ }^{2}$; Salas-Sánchez, J.'; García- \\ Pinillos, F. $^{4}$ \\ ${ }^{1}$ Doctor. Universidad de Jaén. Departamento de Didáctica de la Expresión Corporal (España) \\ platorre@ujaen.es \\ 2 Profesora. Universidad de Jaén (Investigadora). Departamento de Didáctica de la Expresión \\ Corporal (España) elo00003@red.ujaen.es \\ 3 Doctor. Universidad Autónoma de Chile (Chile) jesus.salas@uautonoma.cl \\ 4 Doctor. Universidad de La Frontera, Temuco. Departamento de Educación Física, Deportes y \\ Recreación (Chile) fegarpi@gmail.com
}

Código UNESCO / UNESCO code: 5899 Otras especialidades pedagógicas (Educación Física y Deporte) / Other specialties pedagogical (physical education and sport).

Clasificación Consejo de Europa / Council of Europe classification: 4. Educación Física y deporte comparado / Physical Education and sport compared

Recibido 13 de septiembre de 2019 Received September 13, 2019 Aceptado 22 de junio de 2019 Accepted June 22, 2019

\section{RESUMEN}

El objetivo principal de este estudio fue conocer el perfil evolutivo de la función ejecutiva (FE) y la madurez intelectual (MI) en relación con la condición físicomotora en niños preescolares. Han participado 81 niños, de edades comprendidas entre 3 a 6 años, 44 niños y 37 niñas. Se realizaron pruebas de condición física de fuerza, equilibrio, velocidad, resistencia y tiempo de reacción. Se analizaron las FE y la Ml con los test de Laberintos de Porteus y el test de Goodenough respectivamente. En la evolución de las variables cognitivas y de condición física según la edad de los niños, se observan diferencias significativas entre los grupos de edad. La velocidad de desplazamiento junto con la 
dinamometría manual y el tiempo de reacción son variables que se asocian a las FE y a la MI. Por tanto, existe un paralelismo evolutivo entre el desarrollo de la FE y Ml con el desarrollo de la condición física.

PALABRAS CLAVE: Condición física, función ejecutiva, madurez intelectual, niños, preescolares.

\begin{abstract}
The main aim of this study is to understand the evolutionary profile of the executive function (EF) and the intellectual maturity (IM) in relation to the physical-motor condition in preschool children. A group of 81 children, 3-6 years old, participated in the project (44 boys and 37 girls). Regarding the testing protocol, tests for assessing physical fitness were included (i.e., strength, balance, speed, resistance and reaction time). The EF and the IM were analyzed with the Porteus Maze test and the Goodenough test, respectively. The results showed an age effect on both cognitive variables and physical fitness, with significant differences between those variables between age groups. Additionally, the regression analysis reported a significant association between physical fitness (i.e., sprint, handgrip strength and reaction time) and the FE and the MI. In summary, the results obtained suggest a parallelism between the EF and IM with the development of physical fitness.
\end{abstract}

KEYWORDS: Physical condition, executive function, intellectual maturity, children, preschool.

\title{
INTRODUCCIÓN
}

El estudio del desarrollo infantil ha cobrado importancia en los últimos años. La neurociencia del desarrollo muestra cómo la primeras experiencias biológicas y psicosociales afectan al desarrollo del cerebro (Walker et al. 2011). Sin embargo, la influencia de la actividad física y la condición física (CF) en el desarrollo infantil aún sigue siendo abordada de manera escasa en la investigación científica. En la infancia, la habilidad para ejecutar actos motores es un indicador importante del funcionamiento cognitivo (Campo, 2010). Así el desarrollo motor y cognitivo en la infancia están relacionados entre sí (Poranen-Clark et al. 2015). De hecho, las propiedades arquitectónicas y funcionales globales del cerebro están estrechamente relacionadas con las habilidades locomotoras durante el desarrollo (Isorna, Rial, Felpeto y Rodríguez, 2005; Mierau et al. 2015).

Los acontecimientos mentales y motores comparten el mismo sustrato neural, el sistema neuromotor, o lo que se ha denominado como sistema psicomotriz. La teoría psicomotriz hace hincapié en el papel de liderazgo del sistema motor en la aparición de los eventos mentales (Tan, 2007). Estudios con neuroimagen demuestran el vínculo global entre el rendimiento motor y cognitivo, que indica que las habilidades cognitivas y motoras comparten mecanismos neuronales y aprovechan recursos comunes (Stöckel y Hughes, 2015). 
La CF es un biomarcador importante de la salud desde una edad temprana, siendo el ejercicio físico uno de los principales determinantes de la CF (Ortega, Ruiz, Castillo y Sjöström, 2008). Además, existe una importante conexión entre el crecimiento del cuerpo con la CF (Ortega et al. 2011; Travill, 2011) y con la cognición (Heinonen et al. 2008), destacándose a su vez una relación causal entre la CF y la vitalidad del cerebro, siendo este vínculo importante cuando los efectos de la práctica de ejercicio físico se evalúan con pruebas cognitivas asociadas a la función ejecutiva (FE) (Tomporowski, Davis, Miller y Naglieri, 2008). El término FE se refiere a un constructo multidimensional que engloba una serie de procesos cognitivos necesarios para realizar tareas complejas dirigidas hacia un objetivo (Filippetti, 2011). El desarrollo de las FE durante el período preescolar se puede analizar mediante un marco unitario integrador de componentes parciales como: el trabajo de memoria, la inhibición y la flexibilidad mental o cambio (Miyake, 2000). De los 3 a 5 años se produce un importante desarrollo de las FE asociado con la corteza prefrontal (Garon, Bryson y Smith, 2008).

Los niños con mejor CF presentan una mayor activación cortical y rendimiento cognitivo (Tomporowski et al. 2008). Chang, Labban, Gapin y Etnier (2012) mostraron que la CF era un moderador significativo en la asociación entre la actividad física y el rendimiento cognitivo. Chaddock, Pontifex, Hillman y Kramer (2011) señalan a su vez la importancia de la actividad física y la aptitud aeróbica para la salud del cerebro y la cognición durante el desarrollo.

Por tanto, la evaluación de la CF en niños preescolares debe ser un elemento esencial para el control y seguimiento de la calidad de vida relacionada con la salud, con todas sus implicaciones sanitarias, pero además, debe ser una acción importante para el seguimiento de un desarrollo motor saludable y que otros autores han asociado con un paralelo desarrollo cognitivo (Aberg et al. 2009; Haapala et al. 2013). Los primeros años de vida son fundamentales para el crecimiento físico, cognitivo, motor y el desarrollo socio-emocional, pero la magnitud de la relación entre estos procesos sigue siendo poco clara (Sudfeld et al. 2015). El contar con una perspectiva amplia sobre las características neuropsicológicas de la FE así como de los factores que pueden condicionar su desarrollo, permitirá identificar la secuencia de desarrollo normal de esta función (Lozano y Ostrosky, 2011).

Teniendo en cuenta la anterior información, el objetivo de este estudio es analizar el perfil de evolución de las FE y la madurez intelectual (MI) en relación con la evolución de la CF en niños preescolares.

\section{MATERIAL Y MÉTODO}

\section{PARTICIPANTES}

En este estudio participaron un total de 81 niños de edades comprendidas entre 3 a 6 años, 44 niños y 37 niñas, seleccionados por conveniencia de 4 centros escolares del sur de España tanto de un entorno rural como urbano. Como criterios de inclusión se tuvo en cuenta la escolarización en Educación Infantil y 
no padecer discapacidad física y/o intelectual. Los padres firmaron un consentimiento informado de participación voluntaria de los niños en esta investigación. El estudio fue aprobado por el comité de ética de la Universidad de Jaén.

\section{MATERIALES Y PRUEBAS}

Mediante un cuestionario sociodemográfico realizado ad hoc se recogió información relevante de los padres. Como parámetros antropométricos analizamos la altura (cm), que se midió con un estadiómetro (Seca 222, Hamburgo, Alemania), el peso (kg), que se midió con una báscula (Seca 634, Hamburgo, Alemania), y el índice de masa corporal (IMC) que se obtuvo de la ecuación, $\mathrm{IMC}=$ peso $(\mathrm{kg}) /$ talla $(\mathrm{m})^{2}$. La circunferencia de la cintura se midió mediante una cinta ergonómica (Seca SE201, Alemania). En el análisis de la CF, todos los test seleccionados son validados en población preescolar por Latorre et al. (2015) y representan componentes básicos de la CF como la resistencia, la fuerza, la velocidad, la capacidad de reacción y el equilibrio. Son pruebas sencillas en su comprensión para niños preescolares. Además, se añadió la prueba de dinamometría manual. La resistencia cardiorrespiratoria se evaluó mediante la prueba de $10 \times 20 \mathrm{~m}$, inspirada en la estructura espacial de la prueba de Léger, Mercier, Gadoury y Lambert (1988). En cuanto a la evaluación del equilibrio, se empleó el test de la cigüeña (Johnson y Nelson, 1979). El registro del sprint se realizó a través de una distancia de 20 metros (Fjørtoft, Pedersen, Sigmundsson y Vereijken, 2011). Para analizar el tiempo de reacción (TR) se empleó el Ruler Drop Test (RDT) (Fong, Ng y Chung, 2013). La fuerza de prensión manual se analizó mediante un dinamómetro de mano CAMRY (EH101; Camry, Guangdong Province, China) que presenta un rango 0-90 kg con una precisión $0,1 \mathrm{~kg}$, y un agarre ajustable para tener en cuenta los diferentes tamaños de mano, test validado en población preescolar por Latorre et al. (2017). Por último, se evaluó la fuerza de piernas mediante la prueba de salto horizontal (España-Romero et al. 2010).

La FE se analizó mediante el test de laberintos de Porteus (1965), en éste se presentan 12 laberintos de complejidad creciente con rutas preestablecidas. El test de los laberintos Porteus es una prueba no verbal de inteligencia que permite la evaluación de la capacidad de una persona para realizar un plan. El test presenta adecuados valores de consistencia interna en niños (Krikorian y Bartok, 1998).

La MI se analizó mediante el test del dibujo de la figura humana de Goodenough (GHDT), versión revisada (Harris, 1963). En el GHDT, a los niños se les pide hacer dos dibujos, uno de un hombre y otro de una mujer. La evaluación se centra en los detalles y la proporción general del cuerpo de un dibujo de un hombre (73 datos) y una mujer (71 datos). El GHDT, presenta adecuada fiabilidad y validez en comparación con otras pruebas de inteligencia en niños de 3 a 15 años (Abell, Horkheimer y Nguyen, 1998; Plubrukarn y Theeramanoparp, 2003). En este estudio se utilizó el puntaje crudo promedio de ambos dibujos. 


\section{PROCEDIMIENTO}

Tras la obtención de los permisos oportunos de los centros escolares y del consentimiento informado de los padres, se procedió a la aplicación de los test. En tres sesiones separadas 48 horas, los niños fueron evaluados por un equipo de investigadores adiestrados previamente en los diferentes test. En el primer día se realizaron las pruebas de dinamometría (dos intentos con cada mano), RDT (tres intentos con cada mano), equilibrio (dos intentos con cada pierna) y salto horizontal (dos intentos). El segundo día se registraron la prueba de $20 \mathrm{~m}$ (dos intentos) y $10 \times 20 \mathrm{~m}$ (un intento). En el tercer día se realizaron las pruebas de FE y MI. Previamente a la realización de las pruebas físicas, los niños efectuaron un calentamiento basado en carrera continua y movilidad articular, además se realizaron por parte del equipo investigador unas pruebas de demostración y los niños ejecutaron ensayos de familiarización en las pruebas de dinamometría, RDT, equilibrio y salto horizontal. Se seleccionaron los mejores intentos en cada prueba excepto en la dinamometría manual, RDT y equilibrio que se realizó un promedio de las dos manos y piernas respectivamente con el mejor intento. Cada niño fue evaluado de manera individual. Todas las pruebas se realizaron en las instalaciones deportivas y aulas de los colegios seleccionados. Los niños fueron animados para desarrollar su mejor rendimiento en cada prueba física.

\section{ANÁLISIS ESTADÍSTICO}

Los datos de este estudio se han hallado mediante el programa estadístico SPSS., v.19.0 para Windows, (SPSS Inc, Chicago, USA). El nivel de significación se fijó en $\mathrm{p}<.05$. Los datos se muestran en estadísticos descriptivos de media, desviación típica y porcentajes. Se comprobó la distribución normal de los datos y la igualdad de varianzas mediante pruebas de Kolmogorov-Smirnov y contraste de Levene respectivamente. Las diferencias entre sexos y grupos de edad se analizaron mediante análisis de varianza (ANOVA) con análisis post hoc (Bonferroni). Finalmente se realizó un análisis de correlación Pearson y regresión lineal múltiple entre la MI, FE y la CF ajustado a la edad y al sexo.

\section{RESULTADOS}

En la tabla 1 se exponen las variables sociodemográficas en relación con uno de los padres. 
Tabla 1. Variables sociodemográficas en relación con uno de los padres.

\begin{tabular}{lc}
\hline Estado civil & $\%$ \\
\hline Soltero & 10.8 \\
Casado o en pareja & 85.1 \\
Divorciado/separado & 4.1 \\
\hline Nivel socioeconómico & \\
\hline Bajo & 16.4 \\
Medio & 83.6 \\
\hline Nivel de estudios & \\
\hline Sin estudios & 4.0 \\
Primarios & 25.7 \\
Secundarios & 39.2 \\
Universitarios & 31.1 \\
\hline
\end{tabular}

Ninguna variable socio-demográfica causó diferencias significativas en las variables de CF o cognitivas analizadas. En la tabla 2 se exponen los resultados de edad, variables antropométricas, variables cognitivas y de CF por sexos. Sólo se encuentran diferencias significativas en la circunferencia de la cadera que es mayor en niños.

Tabla 2. Edad, variables antropométricas, variables cognitivas y de condición física por sexos.

\begin{tabular}{|c|c|c|c|}
\hline & $\begin{array}{c}\text { Niño } \\
\text { Media (DT) }\end{array}$ & $\begin{array}{c}\text { Niña } \\
\text { Media (DT) }\end{array}$ & p-valor \\
\hline Edad (meses) & $58.09(13.15)$ & $56.73(13.13)$ & .644 \\
\hline IMC $\left(\mathrm{Kg} / \mathrm{m}^{2}\right)$ & $16.94(2.04)^{\prime}$ & $16.47(1.51)$ & .257 \\
\hline $\begin{array}{l}\text { Circunferencia } \\
(\mathrm{cm})\end{array}$ & $57.98(5.15)$ & $55.51(2.92)$ & .012 \\
\hline Salto horizontal (cm) & $77.74(37.21)$ & 74.97 (32.57) & .728 \\
\hline Velocidad, 20 m (s) & $6.10(1.25)$ & $6.32(1.18)$ & .410 \\
\hline Resistencia (s) & $91.35(22.78)$ & $91.63(22.00)$ & .956 \\
\hline Equilibrio (s) & $10.32(13.26)$ & $11.17(13.79)$ & .778 \\
\hline Dinamometría $(\mathrm{kg})$ & $6.67(2.88)$ & $6.25(2.67)$ & .496 \\
\hline RDT (cm) & 35.52 (11.29) & 37.44 (8.69) & .400 \\
\hline $\mathrm{Ml}(0-73)$ & $6.56(2.27)$ & $7.65(3.05)$ & .077 \\
\hline FE (3-15) & $7.78(2.72)$ & $7.23(2.82)$ & .373 \\
\hline
\end{tabular}

DT: desviación típica. IMC: índice de masa corporal. RDT: ruler drop test. Ml: madurez intelectual. FE: función ejecutiva.

En la tabla 3 se expone la evolución de las variables cognitivas y de CF según la edad de los niños. Se observan diferencias significativas entre todas las variables en relación con los grupos de edad. 
Rev.int.med.cienc.act.fís.deporte - vol. 20 - número 79 - ISSN: 1577-0354

Tabla 3. Evolución de las variables cognitivas y de condición física según la edad de los niños.

\begin{tabular}{|c|c|c|c|c|c|c|c|}
\hline & $\begin{array}{l}3 \text { años } \\
\text { Media (DT) } \\
n=31 \\
a\end{array}$ & $\begin{array}{l}4 \text { años } \\
\text { Media (DT) } \\
n=13 \\
b\end{array}$ & $\begin{array}{l}5 \text { años } \\
\text { Media (DT) } \\
n=19 \\
c\end{array}$ & $\begin{array}{l}6 \text { años } \\
\text { Media (DT) } \\
n=18 \\
d\end{array}$ & P-valor & $\begin{array}{l}\text { Tamaño del } \\
\text { efecto }(\eta 2)\end{array}$ & $\begin{array}{l}\text { Análisis } \\
\text { post-hoc }\end{array}$ \\
\hline Salto horizontal $(\mathrm{cm})$ & $39.57(18.01)$ & $80.50(18.15)$ & $99.21(16.13)$ & $111.33(17.11)$ & $<.001$ & .763 & $\begin{array}{l}a<b, c, d \\
b<c, d\end{array}$ \\
\hline Velocidad, 20 m (s) & $7.29(0.96)$ & $5.93(0.93)$ & $5.46(0.86)$ & $5.30(0.54)$ & $<.001$ & .534 & $a>b, c, d$ \\
\hline Resistencia (s) & $112.45(16.87)$ & 85.95 (17.89) & 75.88 (12.57) & $76.98(11.61)$ & $<.001$ & .561 & $a>b, c, d$ \\
\hline Equilibrio (s) & $1.98(3.26)$ & $5.35(3.57)$ & $15.82(13.77)$ & $24.23(15.36)$ & $<.001$ & .458 & $\begin{array}{l}a<c, d \\
b<c, d\end{array}$ \\
\hline Dinamometría (kg) & $3.83(1.29)$ & $5.73(1.31)$ & $8.50(1.61)$ & $9.45(1.50)$ & $<.001$ & .748 & $\begin{array}{l}a<b, c, d \\
b<c, d\end{array}$ \\
\hline $\begin{array}{l}\text { RDT (cm) } \\
\text { MI (0-73) }\end{array}$ & $\begin{array}{l}40.96(9.52) \\
4.50(2.21)\end{array}$ & $\begin{array}{l}37.80(7.23) \\
8.54(2.33)\end{array}$ & $\begin{array}{l}35.65(10.13) \\
8.76(1.44)\end{array}$ & $\begin{array}{l}28.30(8.48) \\
8.30(1.37)\end{array}$ & $\begin{array}{l}<.001 \\
<.001\end{array}$ & $\begin{array}{l}.221 \\
.546\end{array}$ & $\begin{array}{l}a>d \\
a<b, c, d\end{array}$ \\
\hline FE (3-15) & $5.37(2.01)$ & $7.11(2.15)$ & $8.63(2.03)$ & $10.38(1.64)$ & $<.001$ & .516 & $\begin{array}{l}a<b, c, d \\
b<d\end{array}$ \\
\hline
\end{tabular}

DT: desviación típica. RDT: ruler drop test. MI: madurez intelectual. FE: función ejecutiva. 
En la figura 1, se ilustra el perfil de evolución de las variables de CF y desarrollo cognitivo a lo largo de la edad, se puede observar una evolución paralela en la maduración de la FE, Ml y el resto de pruebas de CF.
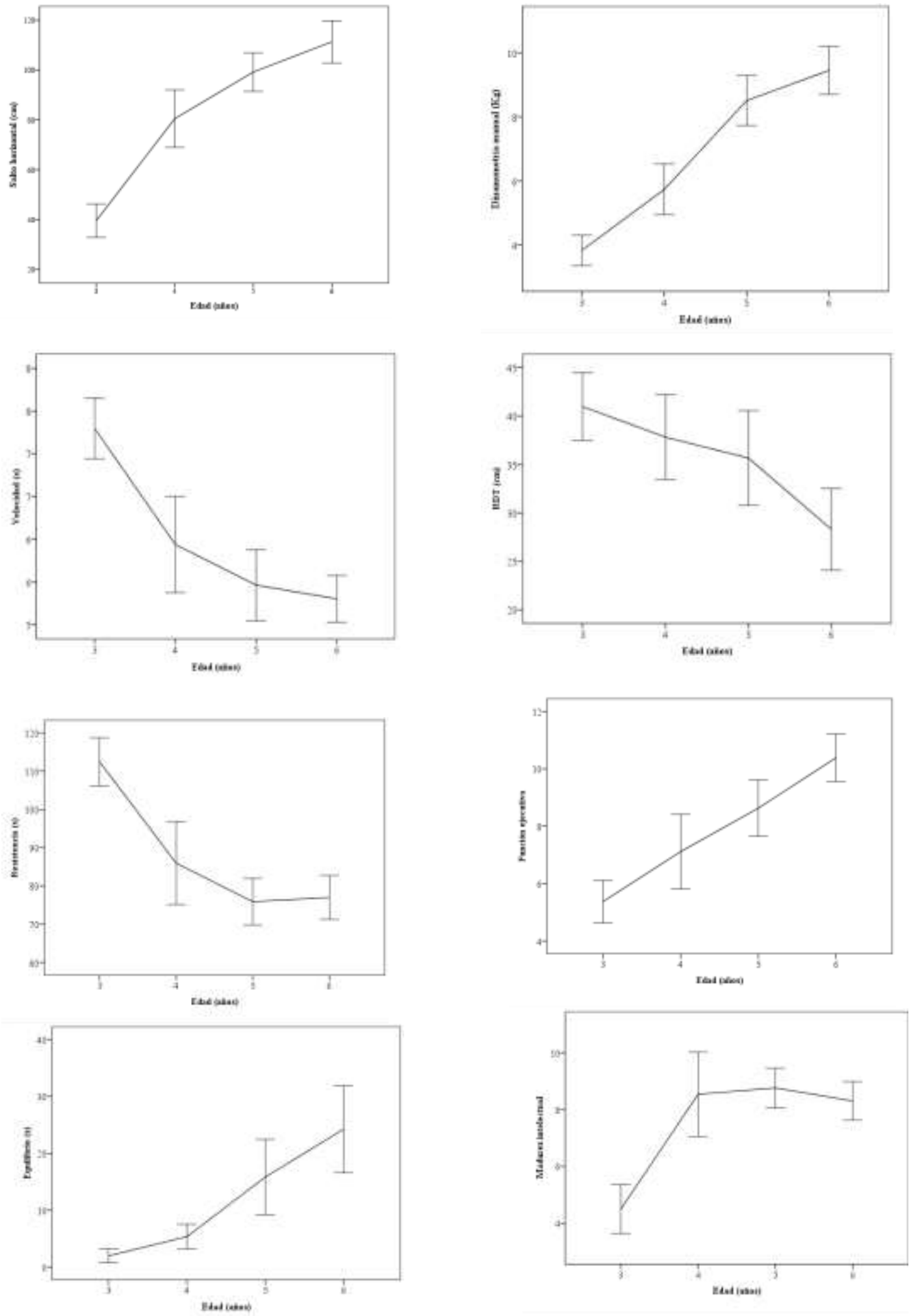

Figura 1. Perfil de evolución de las variables cognitivas y de condición física a lo largo de la edad. 
El análisis de correlación Pearson reveló que las FE y la MI correlacionan de manera positiva entre sí ( $\mathrm{r}=0.256 \mathrm{p}<.027)$, además, las FE correlacionan de manera negativa con el RDT $(r=-0.223, p<.047)$ y la MI positivamente con la dinamometría manual $(r=0.244, p<.035)$ y de manera negativa con el tiempo en recorrer $20 \mathrm{~m}(\mathrm{r}=-0.263, \mathrm{p}<.022)$. En las figuras 2,3 y 4 se exponen los gráficos de dispersión entre la $\mathrm{Ml}$ con la dinamometría manual y el tiempo empleado en recorrer 20 m y el gráfico de dispersión entre las FE y el RDT.

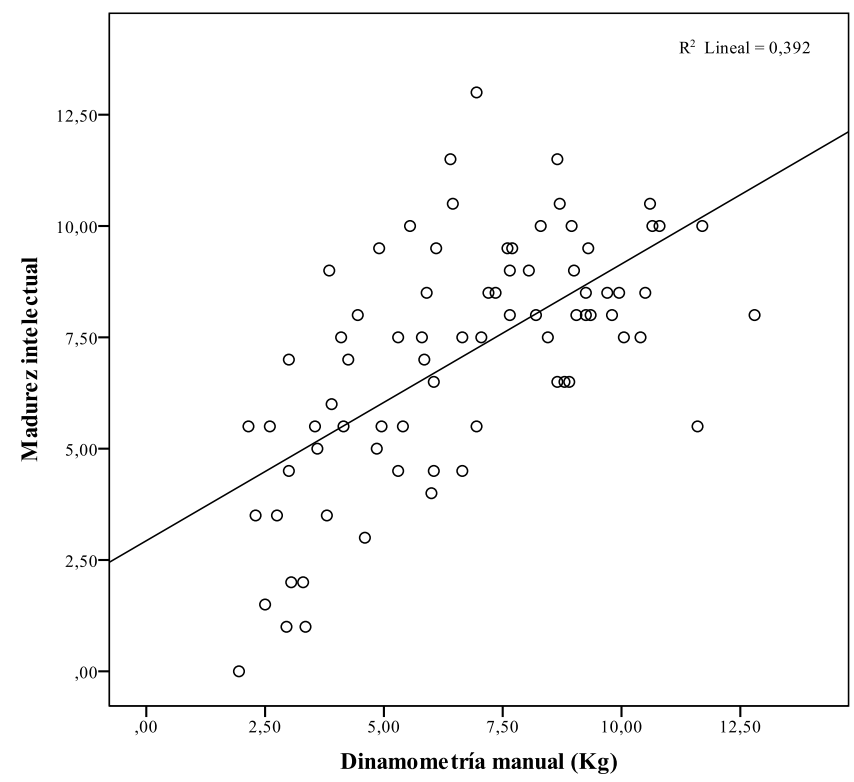

Figura 2. Gráfico de dispersión entre la madurez intelectual y la dinamometría manual.

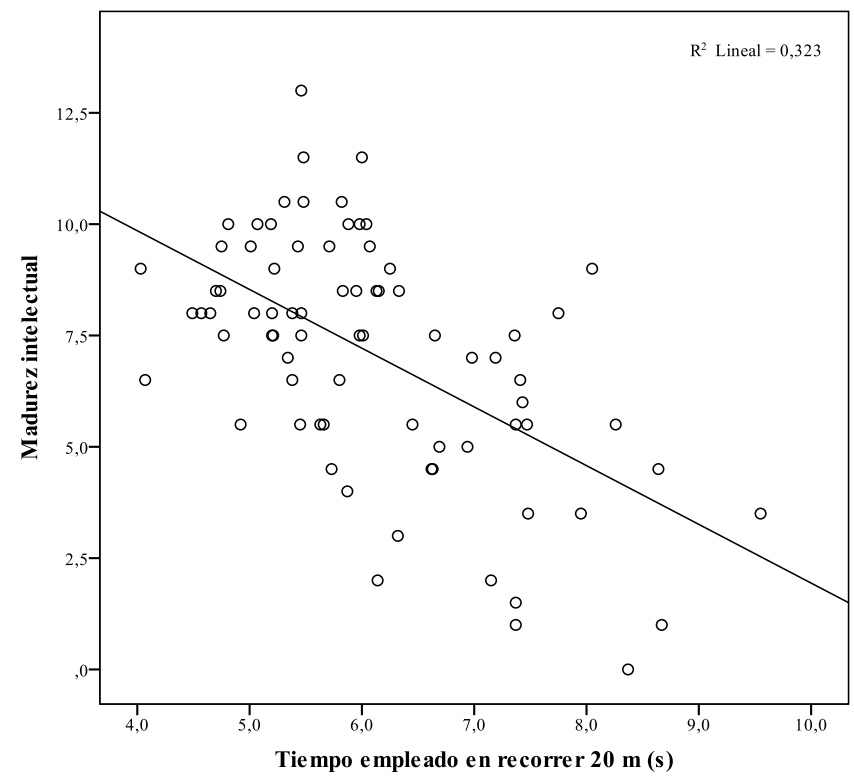

Figura 3. Gráfico de dispersión entre la madurez intelectual y el tiempo empleado en recorrer $20 \mathrm{~m}$. 


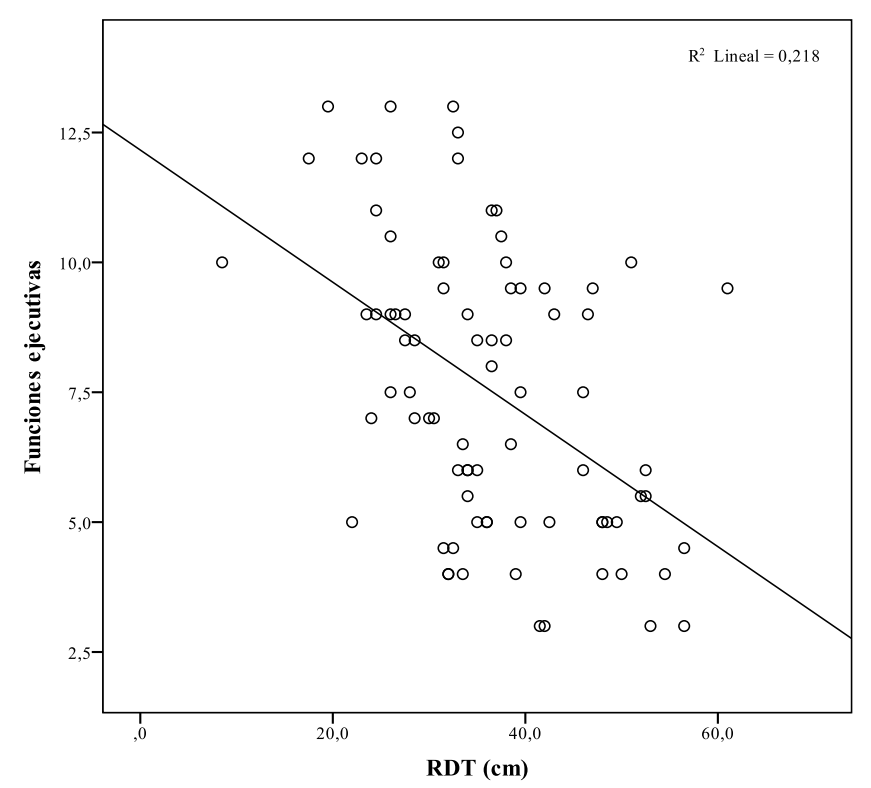

Figura 4. Gráfico de dispersión entre las funciones ejecutivas y el Ruler Drop Test.

\section{DISCUSIÓN}

El objetivo de este estudio ha sido analizar el perfil evolutivo a lo largo de la edad en niños preescolares de la FE, la Ml y la CF, lo que supone un análisis novedoso en la interrelación entre el desarrollo cognitivo y físico. El principal hallazgo de este estudio indica que a lo largo del crecimiento existe un paralelismo evolutivo entre las variables cognitivas y de CF. Además, la velocidad de desplazamiento, la dinamometría manual y el tiempo de reacción son variables asociadas a la MI a las FE en edades preescolares.

En este sentido, Krombholz (2012) muestra una correlación significativa entre el crecimiento físico, el desarrollo de las habilidades motoras y el rendimiento cognitivo en niños preescolares. Igualmente, Wassenberg et al., (2005) señalan un desarrollo paralelo del rendimiento cognitivo y motor específico en los niños durante el desarrollo normal o retrasado así, estructuras cerebrales específicas, como, por ejemplo, los ganglios basales o la corteza frontal y la transmisión de dopamina son compartidos tanto en el rendimiento cognitivo y el rendimiento motor; por lo que el rendimiento motor se relaciona con el rendimiento en varias medidas cognitivas específicas. En el mismo sentido, Krombholz (2006) en un estudio con niños preescolares indica correlaciones significativas entre las medidas de crecimiento y rendimiento físico, el rendimiento motor y cognitivo, la CF, la coordinación y la destreza manual, lo que mejoró en todos los grupos de edad.

En este estudio, la capacidad cardiorrespiratoria no ha mostrado ninguna asociación con las variables cognitivas, sin embargo esta capacidad es quizás la que más relación presenta con la cognición, así la capacidad aeróbica puede estar relacionada con la estructura y función del cerebro en el preadolescente (Chaddock et al. 2010). Métodos con neuroimagen se han empleado para identificar los mecanismos implicados en la relación entre la capacidad aeróbica y la cognición en niños (Chaddock et al. 2011). A su vez, Chaddock et al. (2010) 
sugieren que los déficits en el control cognitivo asociado con una peor capacidad aeróbica pueden relacionarse con diferencias en el volumen de los ganglios basales y menores volúmenes del hipocampo. Los niños que están en buena forma física presentan una mayor activación cortical y rendimiento cognitivo (Tomporowski, 2008) En este sentido, diversas actividades han demostrado mejorar la FE de los niños como, por ejemplo, el trabajo aeróbico, las artes marciales y el yoga (Diamond y Lee, 2011).

Por otro lado, la investigación centrada en el desarrollo de los trastornos como la hiperactividad, el déficit de atención y la apraxia del habla ha sugerido que el rendimiento cognitivo y motor están asociados (Wassenberg et al. 2005). Estudios con sujetos con síndrome de Down muestran una relación entre la competencia motriz y las FE (Schott y Holfelder, 2015). Todos estos resultados indican el desarrollo paralelo de las funciones cognitivas y motoras específicas en niños, tanto con desarrollo normal o retardado.

En este estudio además hemos encontrado una asociación entre la Ml y las FE. Del mismo modo, Rosenthal, Riccio, Gsanger y Jarratt (2006) encontraron relación entre la FE y Ml. Algunos de los aspectos de la FE están involucrados con los sistemas de puntuación del GDHT, así al dibujar una figura humana, un niño debe recordar lo aspectos que pertenecen a una imagen de una persona, como los ojos, la nariz, los oídos, etc., y debe planificar cada parte del dibujo (ojos, oídos, manos, etc.). Por tanto, estas dos pruebas de análisis cognitivo pueden ser un recurso sencillo y práctico para estudios con carácter ecológico, en este caso en el propio contexto escolar.

Durante la infancia y el período preescolar, componentes básicos de las FE se desarrollan, formando una base fundamental que será el escenario para el desarrollo de los mayores procesos cognitivos en la edad adulta (Garon et al. 2008). La identificación temprana de los niños con problemas motores es cada vez más importante en la práctica clínica sobre todo debido a su asociación con el desarrollo cognitivo. El presente estudio proporciona información interesante para el análisis de las relaciones entre el rendimiento cognitivo y motor, que tiene implicaciones para establecer programas de formación e intervención en la primera infancia. Estos hallazgos enfatizan en la identificación temprana de los niños con deficitario rendimiento motor durante los primeros años escolares.

La escuela representa un espacio esencial para la promoción de actividad física (Fradejas, Espada y Garrido, 2016), sin embargo, actualmente en España, a pesar de que la Educación Física y la actividad física forman parte de manera relevante del currículo de Educación Infantil (Real Decreto 1630/2006, de 29 de diciembre) y su reconocimiento es importante en la Comunidad Educativa (Latorre, 2007), la escasez de espacios y recursos materiales adecuados y seguros (Latorre, 2007) y posiblemente una deficiente asignación horaria de las clases de Educación Física en Educación Infantil, provocan que gran parte (aproximadamente un 60\%) del profesorado de Educación Infantil señale que la Educación Física no se trabaja suficientemente (Latorre, 2007). En este sentido, sería importante incorporar un horario específico de Educación Física en Educación Infantil para mejorar los niveles de actividad física y CF y en consecuencia el rendimiento cognitivo de niños de 3 a 6 años. Cambios 
legislativos se hacen necesarios también para promocionar la actividad física en Educación Infantil con el fin de promover la salud, el desarrollo físico y cognitivo de los niños de 3 a 6 años.

Una de las limitaciones de este estudio es su carácter transversal que nos obliga a ser cautos en los resultados obtenidos y en las relaciones causales establecidas. Sin embargo, una fortaleza de esta investigación es que a nuestro conocimiento, escasos estudios han analizado la asociación y evolución de variables cognitivas y de CF en niños preescolares en un entorno escolar, lo cual presenta importantes repercusiones clínicas y educativas.

En conclusión, existe un paralelismo evolutivo entre el desarrollo cognitivo, en concreto la FE y MI, con el desarrollo de la CF, siendo relevante la asociación específica de la dinamometría manual, la velocidad de la carrera y el tiempo de reacción con las variables cognitivas analizadas.

\section{REFERENCIA BIBLIOGRÁFICAS}

Abell, S.C., Horkheimer, R. y Nguyen, S.E. (1998). Intellectual evaluations of adolescents via human figure drawings: An empirical comparison of two methods. Journal of Clinical Psychology, 54(6), 811-815.

https://doi.org/10.1002/(SICI)1097-4679(199810)54:6<811::AIDJCLP8>3.0.CO;2-J

Aberg, M.A.I., Pedersen, N.L., Torén, K., Svartengren, M., Bäckstrand, B., Johnsson, T., ... Kuhn, H.G. (2009). Cardiovascular fitness is associated with cognition in young adulthood. Proceedings of the National Academy of Sciences of the United States of America, 106(49), 20906-20911.

https://doi.org/10.1073/pnas.0905307106

Campo Ternera, L.A. (2010). Importancia del desarrollo motor en relación con los procesos evolutivos del lenguaje y la cognición en niños de 3 a 7 años de la ciudad de Barranquilla (Colombia). Salud Uninorte, Sin mes, 65-76.

https://doi.org/10.21500/19002386.1136

Chaddock, L., Erickson, K.I., Prakash, R.S., Kim, J.S., Voss, M.W., Vanpatter, M., ... Kramer, A.F. (2010). A neuroimaging investigation of the association between aerobic fitness, hippocampal volume, and memory performance in preadolescent children. Brain Research, 1358, 172-183.

https://doi.org/10.1016/.j.brainres.2010.08.049

Chaddock, L., Pontifex, M.B., Hillman, C.H. y Kramer, A.F. (2011). A review of the relation of aerobic fitness and physical activity to brain structure and function in children. Journal of the International Neuropsychological Society, 17(6), 975-985. https://doi.org/10.1017/S1355617711000567

Chang, Y.K., Labban, J.D., Gapin, J.I. y Etnier, J.L. (2012). The effects of acute exercise on cognitive performance: A meta-analysis. Brain Research, 1453, 87-101. https://doi.org/10.1016/..brainres.2012.02.068

Diamond, A. y Lee, K. (2011). Interventions shown to aid executive function development in children 4 to 12 years old. Science, 333(6045), 959-964.

https://doi.org/10.1126/science.1204529 
España-Romero, V., Artero, E.G., Jimenez-Pavón, D., Cuenca-Garcia, M., Ortega, F.B., Castro-Piñero, J., ... Ruiz, J.R. (2010). Assessing healthrelated fitness tests in the school setting: Reliability, feasibility and safety; The ALPHA study. International Journal of Sports Medicine, 31(7), 490497. https://doi.org/10.1055/s-0030-1251990

Filippetti, V.A. (2011). Funciones ejecutivas en niños escolarizados: efectos de la edad y del estrato socioeconómico. Avances en Psicología latinoamericana, 29(1), 98-113.

Fjørtoft, I., Pedersen, A.V., Sigmundsson, H. y Vereijken, B. (2011). Measuring physical fitness in children who are 5 to 12 years old with a test battery that is functional and easy to administer. Physical Therapy, 91(7), 10871095. https://doi.org/10.2522/ptj.20090350

Fong, S., Ng, S. y Chung, L. (2013). Health through martial arts training: Physical fitness and reaction time in adolescent Taekwondo practitioners. Health, 5, 1-5. https://doi.org/10.4236/health.2013.56A3001

Fradejas, E., Espada, M. y Garrido, R. (2016). La Autoconfianza en el Deporte en Edad Escolar. Revista Iberoamericana de Diagnóstico y Evaluación e Avaliação Psicológica, 44(2), 158-171. https://doi.org/10.21865/RIDEP44.2.13

Garon, N., Bryson, S.E. y Smith, I.M. (2008). Executive function in preschoolers: a review using an integrative framework. Psychological Bulletin, 134(1), 31-60. https://doi.org/10.1037/0033-2909.134.1.31

Haapala, E.A., Poikkeus, A.M., Tompuri, T., Kukkonen-Harjula, K., Leppänen P.H., Lindi, V. y Lakka, T.A. (2013). Associations of motor and cardiovascular performance with academic skills in children. Medicine and Science in Sports and Exercise, 46(5), 1016-1024

https://doi.org/10.1249/MSS.0000000000000186

Harris, D.B. (1963). Children's drawings as measures of intellectual maturity. A revision and extension of the Goodenough Draw a Man Test. New York: Harcourt, Brace and World.

Heinonen, K., Räikkönen, K., Pesonen, A.K., Kajantie, E., Andersson, S., Eriksson, J.G., ... Lano, A. (2008). Prenatal and postnatal growth and cognitive abilities at 56 months of age: a longitudinal study of infants born at term. Pediatrics, 121(5), e1325-e1333. https://doi.org/10.1542/peds.2007-1172

Isorna, M., Rial, A., Felpeto, M. y Rodríguez, L. (2017). Evaluación del Impacto del Efecto Relativo de la Edad en el Rendimiento Escolar, Bullying, Autoestima, Diagnostico de TDAH y Consumo de Tabaco en el Paso de Educación Primaria a Secundaria. Revista Iberoamericana de Diagnóstico y Evaluación - e Avaliação Psicológica, 44(2), 92-104.

https://doi.org/10.21865/RIDEP44.2.08

Johnson, B.L. y Nelson, J.K. (1979). Practical measurements for evaluation in physical education. 3. ed. Edina: Burgess Publishing.

Krikorian, R. y Bartok, J.A. (1998). Developmental data for the porteus maze test. The Clinical Neuropsychologist, 12(3), 305-310.

https://doi.org/10.1076/clin.12.3.305.1984

Krombholz, H. (2012). The motor and cognitive development of overweight preschool children. Early Years, 32(1), 61-70.

https://doi.org/10.1080/09575146.2011.599795 
Krombholz, H. (2006). Physical performance in relation to age, sex, birth order, social class, and sports activities of preschool children. Perceptual and Motor Skills, 102(2), 477-484. https://doi.org/10.2466/pms.102.2.477-484

Latorre, P.A. (2007). La motricidad en Educación Infantil, grado de desarrollo y compromiso docente. Revista Iberoamericana de Educación, 43(7), 1-7.

Latorre, P.A., Mora, D., Fernández, M., Salas, J., Moriana, F. y García, F. (2015). Test-retest reliability of a field-based physical fitness assessment for children aged 3-6 years. Nutrición Hospitalaria, 32(4), 1682-1687.

http://dx.doi.org/10.3305/nh.2015.32.4.9486

Latorre, P.Á., Mora, D., Berrios, B., Robles, A., García-Pinillos, F. y Redondo, M. (2017). Handgrip strength is associated with anthropometrics variables and sex in preschool children: A cross sectional study providing reference values. Physical Therapy in Sport, 26, 1-6.

https://doi.org/10.1016/j.ptsp.2017.04.002

Léger, L.A., Mercier, D., Gadoury, C. y Lambert, J. (1988). The multistage 20 metre shuttle run test for aerobic fitness. Journal of Sports Sciences, 6(2), 93-101. https://doi.org/10.1080/02640418808729800

Lozano, A. y Ostrosky F. (2011). Desarrollo de las funciones ejecutivas y de la corteza prefrontal. Revista Neuropsicología, Neuropsiquiatría y Neurociencias, 11(1), 159-172.

Miyake, A., Friedman, N.P., Emerson, M.J., Witzki, A.H., Howerter, A. y Wager, T. D. (2000). The Unity and Diversity of Executive Functions and Their Contributions to Complex "Frontal Lobe" Tasks: A Latent Variable Analysis. Cognitive Psychology, 41, 49-100. https://doi.org/10.1006/cogp.1999.0734

Mierau, A., Felsch, M., Hülsdünker, T., Mierau, J., Bullermann, P., Weiß, B. y Strüder, H.K. (2015). The interrelation between sensorimotor abilities, cognitive performance and individual EEG alpha peak frequency in young children. Clinical Neurophysiology. https://doi.org/10.1016/j.clinph.2015.03.008

Ortega, F.B., Artero, E.G., Ruiz, J.R., España-Romero, V., Jiménez-Pavón, D., Vicente-Rodriguez, G., ... Castillo, M.J. (2011). Physical fitness levels among European adolescents: the HELENA study. British Journal of Sports Medicine, 45(1), 20-29. https://doi.org/10.1136/bjsm.2009.062679

Ortega, F.B., Ruiz, J.R., Castillo, M.J. y Sjöström, M. (2008). Physical fitness in childhood and adolescence: a powerful marker of health. International Journal of Obesity, 32(1), 1-11. https://doi.org/10.1038/sj.ijo.0803774

Plubrukarn, R. y Theeramanoparp, S. (2003). Human Figure Drawing Test: Validity in Assessing Intelligence in Children Aged 3-10 Years. Journal of the Medical Association of Thailand, 86(SUPPL. 3).

Poranen-Clark, T., Von Bonsdorff, M.B., Lahti, J., Räikkönen, K., Osmond, C., Rantanen, T., ...Eriksson, J.G. (2015). Infant motor development and cognitive performance in early old age: the Helsinki Birth Cohort Study. Age (Dordr), 37(3), 1-8. https://doi.org/10.1007/s11357-015-9785-x

Porteus, S.D. (1965). Porteus Maze Test. Fifty years application. New York: Psychological Corporation. https://doi.org/10.1037/t15142-000

Rosenthal, E.N., Riccio, C.A., Gsanger, K.M. y Jarratt, K.P. (2006). Digit Span components as predictors of attention problems and executive functioning in children. Archives of Clinical Neuropsychology, 21(2), 131-139. 
https://doi.org/10.1016/i.acn.2005.08.004

Schott, N. y Holfelder, B. (2015). Relationship between motor skill competency and executive function in children with Down's syndrome. Journal of Intellectual Disability Research, 59(9), 860-872. https://doi.org/10.1111/iir.12189

Stöckel, T. y Hughes, C.M. (2015). The relation between measures of cognitive and motor functioning in 5-to 6-year-old children. Psychological research, 1-12. https://doi.org/10.1007/s00426-015-0662-0

Sudfeld, C.R., McCoy, D.C., Danaei, G., Fink, G., Ezzati, M., Andrews, K.G. y Fawzi, W.W. (2015). Linear growth and child development in low- and middle-income countries: a meta-analysis. Pediatrics, 135(5), e1266e1275. https://doi.org/10.1542/peds.2014-3111

Tan, U. (2007). The psychomotor theory of human mind. The International Journal of Neuroscience, 117(8), 1109-1148.

https://doi.org/10.1080/00207450600934556

Tomporowski, P.D., Davis, C.L., Miller, P.H. y Naglieri, J.A. (2008). Exercise and children's intelligence, cognition, and academic achievement. Educational Psychology Review, 20(2), 111-131. https://doi.org/10.1007/s10648-0079057-0

Travill. A.L. (2011). Correlation between growth and physical fitness of socially disadvantaged girls. South African Journal for Research in Sport, Physical Education and Recreation, 33(3), 139-146. https://doi.org/10.4314/ajpherd.v17i3.68080

Walker, S.P., Wachs, T.D., Grantham-McGregor, S., Black, M.M., Nelson, C.A., Huffman, S.L., ... Richter, L. (2011). Inequality in early childhood: risk and protective factors for early child development. Lancet, 378(9799), 132538. https://doi.org/10.1016/S0140-6736(11)60555-2

Wassenberg, R., Kessels, A.G.H., Kalff, A.C., Hurks, P.P.M., Jolles, J., Feron, F.J.M., ... Vles, J.S.H. (2005). Relation between cognitive and motor performance in 5- To 6-year-old children: Results from a large-scale crosssectional study. Child Development, 76(5), 1092-1103.

https://doi.org/10.1111/j.1467-8624.2005.00899.x

Referencias totales / Total references: 42 (100\%)

Referencias propias de la revista / Journal's own references: $0(0 \%)$

Rev.int.med.cienc.act.fís.deporte - vol. 20 - número 79 - ISSN: 1577-0354 